\title{
Challenges of offering cryo-EM services to National and International users during an extended lockdown period.
}

\author{
Ludo Renault
}

NeCEN / Leiden University, Netherlands

The Netherlands Centre for Electron Nanoscopy $(\mathrm{NeCEN})$ is an open access national facility that provides cryogenic electron microscopy (cryo-EM) services to researchers from academic research institutes and companies, both Dutch and international. NeCEN is located at the Science Faculty of Leiden University in The Netherlands. To serve the users, NeCEN houses a range of instruments for sample preparation, sample characterisation, and high-resolution data collection for electron microscopy as well as state of the art computing resources.

NeCEN provides services to diverse user communities that all have different needs. NeCEN is the high resolution cryo-EM centre in The Netherlands and is central to the Netherlands Electron Microscopy Initiative $(N E M I)$ which is a distributed EM infrastructure regrouping multiple specialized nodes focusing on particular methodologies and expertise across the country.

$\mathrm{NeCEN}$ is also part of the pan-European research infrastructure in structural biology Instruct-ERIC (https://instruct-eric.eu)as well as the European funded project iNEXT-discovery (https://inextdiscovery.eu).

The core service provided to our users is high throughput data collection on two state of the art Titan Krios transmission electron microscopes. NeCEN's experienced microscope operators in combination with the advanced and highly automated hardware allow the user to get most out of their sample and microscope time. An on-the-fly processing and reporting has been developed to ensure the best data quality is collected and the data is uploaded live to our fast-online servers so that the user can download the data live and start processing of the data right away (Alewijnse et al., 2017).

For challenging samples, we offer assistance during buffer optimization and sample screening. For this, a dedicated wet-lab and entry level screening TEM are available on site.

To broaden its portfolio, NeCEN acquired an Aquilos cryo-FIB. With this machine it is now possible to prepare cryo-Lamellae of biological materials for high resolution cryo-Electron Tomography.

During the 2020 SARS-cov2 pandemic, NeCEN maintained a continuous data collection service on the Titan Krios by offering remote access. Coronavirus related projects were prioritized and this resulted into two high impact projects being published (Wolff et al., 2020; Juraszek, Rutten et al., 2021).

NeCEN was directly involved in Juraszek et al. study by providing cryoEM expertise to support vaccine development against SARS-CoV2 by Janssen Pharma company.

In this study, we described the identification of a number of point mutations in the SARS-CoV-2 spike glycoprotein that increase its thermostability and decrease the dynamic behavior of the receptor binding domain in its "down", or closed, conformation while eliminating the need of a stabilizing foldon.

By selecting a minimal combination of this set we created a stable $\mathrm{S}$ trimer in the closed conformation with a 6.4-fold higher expression than the parent construct. The cryo-EM structure reveals a correctly folded, predominantly closed pre-fusion (Fig1) conformation. The high expression levels, melting temperature increase by $\sim 20^{\circ} \mathrm{C}$ and resilience to freeze-thaw will accelerate development of vaccines and serological diagnostics for SARS-CoV-2. 


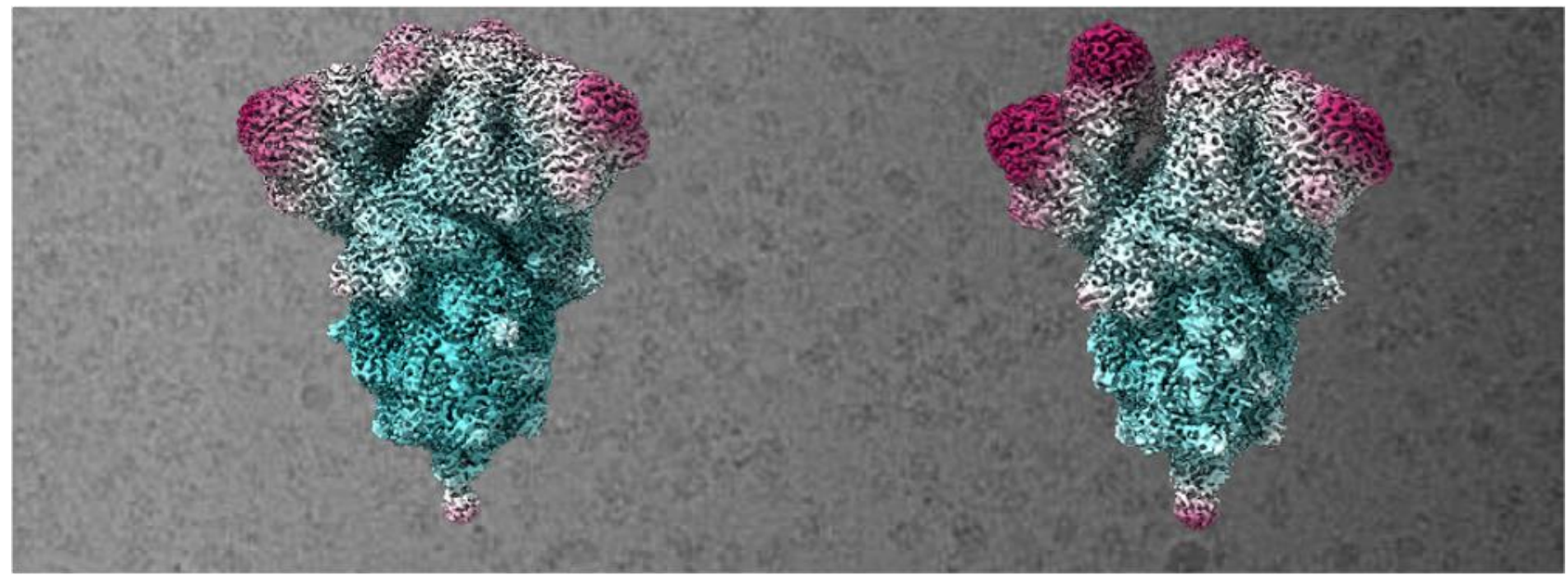

Figure 1. cryo-EM maps of closed (left) and open (right) S-trimer protein colored by local resolution between 2.5 (cyan) and 7.5 (purple) angstroms

References

Alewijnse et al. Best Practices for Managing Large CryoEM Facilities. Journal of Structural Biology doi:10.1016/j.jsb.2017.07.011

Juraszek J, Rutten L, et al. Stabilizing the closed SARS-CoV-2 spike trimer. Nature Communications 12, 244 (2021). doi.org/10.1038/s41467-020-20321-x

Wolff $\mathrm{G}$ et al. A molecular pore spans the double membrane of the coronavirus replication organelle. Science 369, 1395-1398 doi.org/10.1126/science.abd3629 